\title{
Role of Trunk Rehabilitation on Trunk Control, Balance and Gait in Patients with Chronic Stroke: A Pre-Post Design
}

\author{
S. Karthikbabu' ${ }^{1}$, Bhamini K. Rao ${ }^{2}$, N. Manikandan², John M. Solomon², M. Chakrapani ${ }^{3}$, \\ Akshatha Nayak ${ }^{4}$
}

${ }^{1}$ Department of Physiotherapy, Kasturba Medical College, Manipal University, Mangalore, India; ${ }^{2}$ Manipal College of Allied Health
Sciences, Manipal University, Manipal, India; ${ }^{3}$ Kasturba Medical College Clinical Research Centre, Manipal University, Manipal,
India; ${ }^{4}$ MSRMC, Bangalore, India.
Email: karthikbabu78@gmail.com

Received March 2 $2^{\text {nd }}, 2011$; revised April 6 ${ }^{\text {th }}, 2011$; accepted April 20 ${ }^{\text {th }}, 2011$.

\begin{abstract}
Purpose: although proximal stability of the trunk is a prerequisite for balance and gait, to determine the role of trunk rehabilitation on trunk control, balance and gait in patients with chronic stroke is yet unknown. Method: fifteen subjects (post-stroke duration $(3.53 \pm 2.98)$ years) who had the ability to walk 10 meters independently with or without a walking aid; scoring $\leq 21$ on Trunk Impairment Scale (TIS), participated in a selective trunk muscle exercise regime, consisting of 45 minutes training per day, four days a week, and for four weeks duration in an outpatient stroke rehabilitation centre. Results: the overall effect size index for trunk rehabilitation was 1.07. This study showed large effect size index for Trunk Impairment Scale (1.75), Berg Balance Scale (1.65) than for gait variables (0.65). After trunk rehabilitation, there was a significant improvement for gait speed $(p=0.015)$, cadence $(p=0.001)$ and gait symmetry $(p=$ $0.019)$ in patients with chronic stroke. In addition, all the spatial gait parameters had a significant change post-intervention. There was no significant change in temporal gait parameters with the exception of affected single limb support time. The level of significance was set at $p<0.05$. Conclusion: the exercises consisted of selective trunk movement of the upper and the lower part of trunk had shown larger effect size index for trunk control and balance than for gait in patients with chronic stroke. Future randomized controlled studies incorporating large sample size would provide insight into the effectiveness and clinical relevance of this intervention.
\end{abstract}

Keywords: Selective trunk activity, Trunk rehabilitation, Stroke, Trunk control, Balance, Gait

\section{Introduction}

The sensory-motor impairment of trunk interferes with the functional performance after stroke [1,2]. Contrary to common belief, the trunk muscles are impaired on both the sides of the body in patients with stroke [3,4]. Studies reported the weakness of trunk flexor-extensor and bilateral trunk rotator muscles by means of isokinetic dynamometer muscle strength testing in patients with chronic stroke, when compared to that of age matched healthy controls [5,6]. A study using Trunk Impairment Scale (TIS) also found that selective movements of the upper and the lower trunk are impaired in chronic stroke [7].

The trunk being the central key point of the body, proximal trunk control is a prerequisite for distal limb movement control, balance and functional activities.
Trunk control is the ability of the trunk muscles to allow the body to remain upright, adjust weight shift, and performs selective movements of the trunk so as to maintain the center of mass within the base of support during static and dynamic postural adjustments [8-10]. A study on electromyography analysis observed that the anticipatory postural adjustment of trunk muscles activity is impaired in patients with stroke [11]. A cross sectional study by Verheyden et al. [12] demonstrated that trunk control is related to measures of balance, gait and functional ability in patients with stoke. Trunk control has also been identified as an important early predictor of functional outcome after stroke [13-15].

Most literature concerning rehabilitation after stroke focuses on the hemiplegic upper and lower limbs while 
the trunk receives little attention. Unlike limb muscles, the abdominal muscles need a stable origin to act efficiently, that is the pelvis, the thorax or the central aponeurosis depending upon part of trunk that is moved. Counter rotation between the upper and lower trunk is the mobility over stability task which is essential for all the functional movements. The rotation of the trunk muscle activity is not unilateral, but requires static holding of contra-lateral muscles to stabilize the central aponeurosis, so allowing the antagonist shorten and draws one side the pelvic or thorax forwards. In addition, the trunk rotators cannot function efficiently when their origin and insertion are approximated, as the spine is flexed [8]. A recent study on dynamic posturographic analysis stated that trunk movements in person with stroke are executed by upper trunk with very minimal anterior tilt of the pelvis i.e. mobility over stability skill is impaired [16]. Therefore, selective trunk muscle exercises are indeed related to clinical practice in patients with stroke. A randomized trial that added 10 hours additional trunk exercises to regular rehabilitation had a beneficial effect in improving trunk control, particularly the dynamic sitting postural control in sub-acute stroke [17]. A study by Mudie et al. [18] found that training the patient in the awareness of trunk position could improve sitting weight symmetry in sub-acute stroke. Although proximal trunk control is a prerequisite for improving balance and weight symmetry, no studies have reported the role of physiotherapy in treating the trunk for patients with chronic stroke. To the best of our knowledge, this study is the first of its kind which attempted to determine the role of selective trunk muscle exercise training on trunk control, balance and gait in patients with chronic stroke.

\section{Methods}

\subsection{Subjects}

A pilot quasi-experimental design was conducted in the neurological rehabilitation centre of the outpatient stroke unit, of a multi-specialty teaching hospital. The study protocol was approved by the Ethics and Scientific Committee of the Institution, Manipal University, India. The purpose of the study was explained to the subjects with chronic stroke and written informed consent was obtained seeking their active participation. The participants who consented were screened for inclusion and exclusion criteria. Subjects with chronic stroke of at least six months duration were included in the study if they met the following criteria: 1) first onset of unilateral supratentorial hemorrhagic or ischemic stroke; 2) able to understand and follow simple verbal instruction, scoring at least 24 out of 30 on Mini Mental State Examination; 3) ability to walk at least 10 meters distance independently, with or without a walking aid; 4) currently not receiving any other type of therapeutic intervention; 5) scoring less than 21 out of 23 on Trunk Impairment Scale [7]. Patients were excluded if they had a neurological disease affecting balance other than stroke such as Parkinson's disease and/or Vestibular lesion, and a history of diagnosed musculoskeletal disorders of the trunk and/or lower extremities affecting the motor performance.

\subsection{Intervention}

All the treatment sessions were delivered by physiotherapist who was not involved in measuring the outcomes. All the patients received exercises consisting of selective movements of the upper and the lower part of the trunk in supine and sitting. The supine exercises involved the pelvic bridge, the unilateral bridge, the flexion rotation of the upper and lower trunk. Sitting exercises included selective flexion extension of the lower trunk; lateral flexion of the upper and lower trunk; rotation of the upper and the lower trunk; forward and lateral reach. The trunk exercises were initiated with moderate assistance to obtain proper quality of movement and progressed to a state of no assistance. The number of repetitions and intensity of the exercise were determined by the physiotherapist based on the patient's performance. These exercises were performed with adequate rest periods in between. The intensity of the exercises was increased by introducing one or several of the following changes: 1) reducing the base of support; 2) increasing the lever arm; 3) advancing the balance limits; 4) increasing the hold time. The selective trunk muscle exercise training was practiced for 45 minutes a day, four days a week and for four weeks duration.

The supine exercises were as follows: the pelvic bridge was performed by lifting the pelvis off the plinth from crook-lying. The exercise intensity was further increased by flexing the uninvolved upper limb. The unilateral pelvic bridge was performed by lifting the uninvolved leg off the plinth while maintaining the pelvic bridge position. Upper trunk rotation was executed by bringing clasped hands on either side. The patient was asked to perform a task-specific reach-out for an object kept above the hip by a flexion rotation of the upper trunk. Lower trunk rotation was performed by moving the knees on either side from crook-lying. The flexion rotation of the lower trunk was achieved by bringing the knees diagonally towards the shoulder.

The sitting exercises were as follows: the patient was seated on the functional re-education plinth with hips and knee bent at $90^{\circ}$ angles and the feet kept flat on the floor. Selective flexion extension of the lower trunk was performed by ante-flexion and retro-flexion of the lower 
part of the trunk. Upper trunk lateral flexion was executed by initiating movement from the shoulder girdle so as to bring the elbow towards the plinth. Lower trunk lateral flexion was achieved by initiating movement from the pelvic girdle so as to lift the pelvis off the plinth and bring towards the rib cage. Upper and lower trunk rotations were performed by moving both the shoulders and knees forwards and backwards respectively. A forward reach was performed by asking the patient to reach a fixed point at shoulder height by forward flexing the trunk at the hips. Furthermore, progression was made by a forward diagonal reach at shoulder height. A lateral reach was performed by reach out for a fixed point at shoulder height so as to elongate the trunk on the weight bearing side and shorten the trunk on the non-weight bearing side.

\subsection{Outcome Measures}

Trunk Impairment Scale (TIS), Berg Balance Scale (BBS) and 10 meters distance walk test were the outcome tools to measure the trunk control, balance and gait parameters respectively. All the outcome measures were collected by physiotherapist who was not involved in conducting the study intervention. The TIS is a 4-point ordinal scale which evaluates static sitting balance, dynamic sitting balance and coordination. In earlier studies it had been documented for its reliability, validity and responsiveness $[19,20]$. The BBS is a 14-item scale that quantitatively assesses balance in stroke patients. It had been documented for its construct validity, concurrent validity, and predictive validity, inter rater reliability, test-retest reliability, and internal consistency in earlier research [21-24].

Patients were asked to walk on a 10 meters distance walkway with long lasting dye applied at their feet. The motion-picture was recorded by a camcorder so as to capture the movement sequences of the lower limbs for each phases of the gait cycle in a sagittal plane. The middle six meter distance was considered for analysis to permit acceleration and deceleration effects. The average of three 10-meter distance walk trials was considered to measure gait parameters [25]. Gait speed was calculated by dividing the distance walked by the time duration. Cadence was calculated by counting the number of steps placed on the floor in a minute. Spatial gait parameters such as step length and stride length were calculated measuring the distance between foot prints using a standard measuring tape. Temporal gait parameters such as single limb support time and double limb support time were processed using Adobe Premiere $1.5^{\circledR}$ software. It is motion-picture analyzer which provides 25 frames in a second, each frame lasts for 0.04 milliseconds. The temporal parameters were obtained by computing the time spent in all the single and double limb stance phases of affected and unaffected side. Temporal gait asymmetry was calculated by finding the ratio between the affected and the unaffected single limb stance.

\section{Analysis and Results}

\subsection{Statistical Analysis}

Descriptive measures are summarized as mean $\pm \mathrm{SD}$ or percentage, as mentioned. The paired t-test was used to compare the difference between pre-intervention and post-intervention scores. The level of significance was set at $p<0.05$. The analysis was performed using the SPSS version 11.5. Effect size index (d) was calculated for each of the dependent measures using the formula $\mathrm{d}=$ d' $\sqrt{2}$. The effect size (d') was computed using the formula $\mathrm{d}^{\prime}=d / \mathrm{s}_{\mathrm{d}}$ where $d$ is the mean difference scores, and $\mathrm{S}_{\mathrm{d}}$ is the standard deviation of the difference scores. The overall effect size index was determined by averaging the trunk control, balance and gait effect size indices. Effect size index was then defined using Cohen's classification of effect size index $(d)$, where small $d=0.20$, medium $\mathrm{d}=0.50$ and large $\mathrm{d}=0.80$ [26].

\subsection{Results}

Of the 42 patients screened for study eligibility, a total of 20 patients were included in the study, of which 15 completed the study intervention. Table 1 shows the participants' demographic characteristics. The overall effect size index for all the outcome measures and power of the study were 1.07 and $75 \%$ respectively. The effect size index for trunk control (1.75) and balance (1.65) were large compared to gait $(0.65)$. In order to minimize type II error (power of $80 \%$ ), 17 patients would be required. Table 2 shows effect size index, power and number of patients needed to reach a power of 0.80 . Table 3 shows the participants' outcome variables at base line and posttreatment.

Table 1. Demographic characteristics (mean \pm SD or $n(\%)$ ).

\begin{tabular}{ll}
\hline Item & $\boldsymbol{N}=\mathbf{1 5}$ \\
\hline Age (years) & $50 \pm 11.87$ \\
Post-stroke duration (years) & $3.53 \pm 2.98$ \\
Gender (male/female) & $11(73 \%) / 4(27 \%)$ \\
Hemiplegic side (right/left) & $5(33 \%) / 10(67 \%)$ \\
Stroke lesion (ischemic/hemorrhage) & $9(60 \%) / 6(40 \%)$ \\
\hline
\end{tabular}

Values are expressed as mean $\pm \mathrm{SD}$ or $n(\%)$. 
Table 2. Effect size index and power calculations for outcome measures.

\begin{tabular}{|c|c|c|c|c|c|c|c|}
\hline & \multirow{2}{*}{$\begin{array}{c}\text { Trunk Control } \\
\text { TIS }^{@}\end{array}$} & \multirow{2}{*}{$\begin{array}{c}\text { Balance } \\
\text { BBS }^{\#}\end{array}$} & \multicolumn{4}{|c|}{ Gait } & \multirow{2}{*}{$\begin{array}{c}\text { Trunk control } \\
+ \text { Balance + } \\
\text { Gait Average }\end{array}$} \\
\hline & & & Speed & Cadence & $\begin{array}{c}\text { Stride } \\
\text { Length }\end{array}$ & Average & \\
\hline Effect size index (d) & $1.75(\mathrm{~L})$ & $1.65(\mathrm{~L})$ & $0.65(\mathrm{M}-\mathrm{L})$ & $0.55(\mathrm{M})$ & $0.74(\mathrm{~L})$ & $0.65(\mathrm{M}-\mathrm{L})$ & $1.07(\mathrm{~L})$ \\
\hline Power $(n=15)^{*}$ & 1.00 & 1.00 & 0.40 & 0.30 & 0.50 & 0.40 & 0.75 \\
\hline No. of patients need for power of $0.80^{*}$ & 6 & 7 & 39 & 54 & 29 & 39 & 17 \\
\hline
\end{tabular}

${ }^{\circledR}$ Trunk Impairment Scale (TIS); ${ }^{*}$ Berg Balance Scale (BBS); ${ }^{*} \alpha=0.05 ;$ M (Medium d $\left.=0.50\right) ;$ L $($ Large $d=0.80)$.

Table 3. All the outcome measures at pre and post interventions levels.

\begin{tabular}{lccc}
\hline Outcome measures & Pre-intervention $(\boldsymbol{n}=\mathbf{1 5})$ & Post-intervention $(\boldsymbol{n}=\mathbf{1 5})^{\boldsymbol{p}_{\text {value }}^{*}}$ \\
\hline Trunk Impairment Scale (0 - 23) & $17.83 \pm 1.62$ & $19.4 \pm 1.95$ & 0.001 \\
Berg Balance Scale (0 - 56) & $41 \pm 7.81$ & $47 \pm 6.72$ & 0.001 \\
Gait speed (meter per second) & $0.47 \pm 0.19$ & $0.54 \pm 0.23$ & 0.009 \\
Cadence (steps per minute) & $76.06 \pm 18.41$ & $81.40 \pm 20.75$ & 0.001 \\
Spatial parameters (centimeter) & & & 0.011 \\
Affected stride length & $66.1 \pm 17.78$ & $74.14 \pm 25.08$ & 0.007 \\
Unaffected stride length & $65.92 \pm 17.86$ & $73.7 \pm 20.88$ & 0.011 \\
Affected step length & $35.92 \pm 11.7$ & $40.56 \pm 13.52$ & 0.006 \\
Unaffected step length & $30.11 \pm 10.12$ & $33.82 \pm 11.23$ & \\
Temporal parameters (millisecond) & & & $0.44 \pm 0.13$ \\
Affected single limb support & $0.38 \pm 0.07$ & $0.54 \pm 0.16$ & 0.029 \\
Unaffected single limb support & $0.54 \pm 0.14$ & $0.32 \pm 0.13$ & 0.817 \\
Affected double limb support & $0.36 \pm 0.16$ & $0.42 \pm 0.15$ & 0.183 \\
Unaffected double limb support & $0.46 \pm 0.16$ & $1.23 \pm 0.14$ & 0.223 \\
Temporal gait asymmetry & $1.42 \pm 0.08$ & 0.019 \\
\hline
\end{tabular}

"Values are expressed as mean $\pm \mathrm{SD}$; ${ }^{*}$ statistically significant at $p$ value $<0.05$.

\section{Discussion and Conclusions}

\subsection{Discussion}

The overall effect size index (d) of this study was 1.07. Selective trunk muscle training showed a task specific effect on trunk control $(\mathrm{d}=1.75)$ and also a carry-over effect on balance $(d=1.65)$ and gait variables $(d=0.65)$. The study findings warrant caution when interpreting and generalizing the results. The study had a limited number of participants recruited from a single geographical location and had no control group. Without a control group, it is cautioned to state whether the gains were truly due to the selective trunk muscle exercises. However, the participants with chronic stroke $(3.53 \pm 2.98$ years $)$ had very little room for spontaneous recovery, and were not receiving any other therapeutic intervention at the time beginning of study. Therefore, we may substantiate our claim that improved trunk control and balance were due to trunk training.

Motor learning literature suggests that training needs to be specific to the task that the person needs to do. The large effect size index (d) observed in the trunk control (1.75), support our study assumption. A study by Verheyden et al. [17] also favors this hypothesis. In their study, 10 hours of additional task specific trunk exercises along with regular rehabilitation had improved the trunk control in patients with sub-acute stroke. A study by Mudie et al. [18] found that training the patient in the awareness of trunk position could improve weight symmetry in sitting after the early phase of the stroke.

We observed a change score of 6 BBS points increase with trunk rehabilitation in our study. A study by Liaw et al. [27] found that a change of $7 \mathrm{BBS}$ points in patients with chronic stroke is necessary in order to be $90 \%$ confident of a genuine change. After trunk rehabilitation, the majority of the participants scored better in dynamic double and single stance items of the BBS, but individual items of the BBS were not examined. Furthermore, the majority of participants surpassed the score, beyond 45 on the BBS, and decreasing their risk of falls [28]. Rationale for the gains in balance when all the training was done in supine and sitting is given below. Selective trunk 
muscle exercises in supine position include the use of lower limb muscles also, which could account for change in BBS results. Experts in the field of neurological rehabilitation have addressed the trunk as the central key point of the body, and the control of movement proceeds from proximal to distal body regions. Motor control literature suggests that if an improved level of proximal trunk control gains were attained, a better distal limb control might be anticipated during balance and functional mobility. A recent cross sectional study by Verheyden et al. [12] favors this hypothesis. In their study, there was a positive association found between the trunk performance and the balance after sub-acute stroke. Furthermore, studies by Dean et al. [29,30] found that, there was an improvement of standing balance following dynamic sitting balance training in patients with stroke.

Despite the study result was statistically significant for gait speed $(0.07 \mathrm{~m} / \mathrm{s})$ post-intervention, the minimum detectable change (MDC) value was relatively much less when compared to $0.16 \mathrm{~m} / \mathrm{s}$ found in a study by Tilson $e t$ al. [31]. According to Tilson et al., patients with subacute stroke who increase gait speed $\geq 0.16 \mathrm{~m} / \mathrm{s}$ are more likely to experience a meaningful improvement in disability level than those who do not. This may explain the fact that gait speed MDC change may warrant caution to affirm about the clinically meaningful difference. Importantly, subjects with sub-acute stroke may still have the potential for spontaneous recovery, while our study participants had very little room for spontaneous recovery. Goldie et al. [32] reported that the two determinants of gait speed, cadence and step length, decrease after stroke. A change in cadence i.e. 5 steps increase, may be the potential contributing factor for increased speed in our study participants. In addition, the significant change seen in all the spatial parameters may further support for the improved gait speed. Furthermore, the probable reason for change in gait speed may be due to improved trunk control with trunk rehabilitation. Therefore, if an improved level of proximal trunk control was attained, a better the distal lower extremity mobility might be anticipated such as that involved in walking.

After stroke, a decreased time in single limb support of the affected limb may indicate difficulty in balancing or bearing full body weight on the affected limb. Asymmetric gait in chronic hemiplegia is characterized by longer time spent in affected single limb stance than unaffected single limb stance [33]. Recent studies on posturographic analysis observed that stroke patients tend to avoid shifting their center of pressure towards the hemiplegic side in sitting [34] and standing [35]. A study on involving the analysis of trunk kinematics during walking found that pelvic movements were unstable and asymmetrical in patients with stroke [36]. Consequently, there may be a possibility exists that selective trunk muscle exercise training may enhance symmetrical pelvic movements, thus better weight shifting towards hemiplegic limb during walking. With trunk rehabilitation, increased time spent in affected limb support stance may be reason for the gait symmetry improvement. A study by Trueblood et al. [37] favors this hypothesis. In their study, PNF based resisted anterior elevation and posterior depression of pelvic movements for trunk muscles had resulted better temporal gait symmetry in patients with sub-acute stroke.

The study findings are of clinical importance since they indicate an improved trunk control, balance and gait owing to an inclusion of selective trunk muscle exercise regime in the comprehensive rehabilitation of patients with chronic stroke. Future randomized controlled studies with a large number of patients are needed to confirm our study results. There was no follow up of the patients in order to find out if improvement was carried over, and the functional status of the patients was not assessed following intervention. Future randomized controlled trials should assess the long term effects of trunk rehabilitation on the level of balance self-efficacy and community reintegration after chronic stroke.

\subsection{Conclusions}

Selective trunk muscle exercise regime has an overall large effect size index. This study showed a large effect size index for trunk control and balance than for gait. Treatment of hemiplegic lower limb along with the selective trunk exercise regime may result in a large effect size index for the gait. Future randomized controlled studies incorporating trunk rehabilitation with large sample size would provide insight into the effectiveness and clinical relevance of this intervention.

\section{Acknowledgements}

The authors thank all the patients for their active participation in this study. We are grateful to Mr. Devanand, Manual Therapist, HOSEMET Hospital, Bangalore, India for his valuable advice and suggestion in drafting the manuscript.

\section{REFERENCES}

[1] S. Ryerson, N. Byl, D. Brown, R. Wong and J. Hidler. "Altered Trunk Position Sense and Its Relation to Balance Functions in People Post-stroke," Journal of Neurological Physical Therapy, Vol. 32, No. 1, 2008, pp. 14-20.

[2] M. Karatas, N. Cetin, M. Bayramoglu and A. Dilek, "Trunk Muscle Strength in Relation to Balance and Functional Disability in Unihemispheric Stroke Patients," American Journal of Physical Medicine and Rehabilitation, Vol. 83, No. 2, 2004, pp. 81-87.

doi:10.1097/01.PHM.0000107486.99756.C7 
[3] T. Fujiwara, S. Sonoda, Y. Okajima and N. Chino, "The Relationships between Trunk Function and the Findings of Transcranial Magnetic Stimulation among Patients with Stroke," Journal of Rehabilitation Medicine, Vol. 33, No. 6, 2001, pp. 249-255. doi:10.1080/165019701753236428

[4] T. Tsuji, M. Liu, K. Hase and N. Chino, "Trunk Muscles in Persons with Hemiparetic Stroke Evaluated with Computed Tomography," Journal of Rehabilitation Medicine, Vol. 35, No. 4, 2003, pp. 184-188. doi:10.1080/16501970306126

[5] S. Tanaka, K. Hachisuka and H. Ogata, "Muscle Strength of the Trunk Flexion-Extension in Post-Stroke Hemiplegic Patients," American Journal of Physical Medicine and Rehabilitation, Vol. 77, No. 4, 1998, pp. 288-290. doi:10.1097/00002060-199807000-00005

[6] S. Tanaka, K. Hachisuka and H. Ogata, "Trunk Rotatory Muscle Performance in Post-Stroke Hemiplegic Patients," American Journal of Physical Medicine and Rehabilitation, Vol. 76, No. 5, 1997, pp. 366-369.

doi:10.1097/00002060-199709000-00003

[7] G. Verheyden, A. Nieuwboer, H. Feys, V. Thijs, K. Vaes and W. de Weerdt, "Discriminant Ability of the Trunk Impairment Scale: A Comparison between Stroke Patients and Healthy Individuals," Disability Rehabilitation, Vol. 27, No. 17, 2005, pp. 1023-1028. doi:10.1080/09638280500052872

[8] P. M. Davis, "Problems Associated with the Loss of Selective Trunk Activity in Hemiplegia," In: P. M. Davis, Ed., Right in the Middle. Selective Trunk Activity in the Treatment of Adult Hemiplegia, Springer, New York, 1990, pp. 31-65.

[9] S. Ryerson and K. Levit, "Functional Movement: A Practical Model for Treatment," In: S. Ryerson and K. Levit, Ed., Functional Movement Reeducation. A Contemporary Model for Stroke Rehabilitation, Churchill Livingstone, Philadelphia, 1997, pp. 1-14.

[10] S. Edwards, "An Analysis of Normal Movement as the Basis for the Development of Treatment Techniques," In: S. Edwards, Ed., Neurological Physiotherapy. A Problem-Solving Approach, Churchill Livingstone, Philadelphia, 1996, pp. 5-40.

[11] R. Dickstein, S. Shefi, E. Marcovitz and Y. Villa, "Anticipatory Postural Adjustments in Selected Trunk Muscles in Post-Stroke Hemiparetic Patients," Archives of Physical Medicine and Rehabilitation, Vol. 85, No. 2, 2004, pp. 261-273. doi:10.1016/j.apmr.2003.05.011

[12] G. Verheyden, L. Vereeck, S. Truijen, M. Troch, I. Herregodts, C. Lafosse, et al., "Trunk Performance after Stroke and Relationship with Balance, Gait and Functional Ability," Clinical Rehabilitation, Vol. 20, No. 5, 2006, pp. 451-458. doi:10.1191/0269215505cr955oa

[13] E. Duarte, E. Marco, J. Muniese, R. Belmonte, P. Diaz, M. Tejero, et al., "Trunk Control Test as a Functional Predictor in Stroke," Journal of Rehabilitation Medicine, Vol. 34, No. 6, 2002, pp. 267-272. doi:10.1080/165019702760390356

[14] C. L. Hsieh, C. F. Sheu, I. P. Hsueh and C. H. Wang,
"Trunk Control as an Early Predictor of Comprehensive Activities of Daily Living Function in Stroke Patients," Stroke, Vol. 33, 2002, pp. 2626-2630. doi:10.1161/01.STR.0000033930.05931.93

[15] G. Verheyden, A. Nieuwboer, L. de Wit, H. Feys, B. Schuback, I. Baert, et al., "Trunk Performance after Stroke: An Eye Catching Predictor of Functional Outcome," Journal of Neurology Neurosurgery and Psychiatry, Vol. 78, No. 7, 2007, pp. 694-698. doi:10.1136/jnnp.2006.101642

[16] S. Messier, D. Bourbonnais, J. Desrosiers and Y. Roy, "Dynamic Analysis of Trunk Flexion after Stroke," Archives of Physical Medicine and Rehabilitation, Vol. 85, No. 10, 2004, pp. 1619-1624. doi:10.1016/j.apmr.2003.12.043

[17] G. Verheyden, L. Vereeck, S. Truijen, M. Troch, C. LaFosse, W. Saeys, et al., "Additional Exercises Improve Trunk Performance after Stroke: A Pilot Randomized Controlled Trial," Neurorehabilitation and Neural Repair, Vol. 23, No. 3, 2008, pp. 281-286. doi: $10.1177 / 1545968308321776$

[18] M. H. Mudie, U. Winzeler-Mercy, S. Radwan and L. Lee, "Training Symmetry of Weight Distribution after Stroke: A Randomized Controlled Pilot Study Comparing Task-related Reach, Bobath and Feedback Training Approaches," Clinical Rehabilitation, Vol. 16, No. 6, 2002, pp. 582-592. doi:10.1191/0269215502cr527oa

[19] G. Verheyden, A. Nieuwboer, J. Mertin, R. Preger, C. Kiekens and W. de Weerdt, "The Trunk Impairment Scale: A New Tool to Measure Motor Impairment of the Trunk after Stroke," Clinical Rehabilitation, Vol. 18, No. 3, 2004, pp. 326-334. doi:10.1191/0269215504cr733oa

[20] G. Verheyden, A. Nieuwboer, A. van de Winckel and W. de Weerdt, "Clinical Tools to Measure Trunk Performance after Stroke; a Systematic Review of the Literature," Clinical Rehabilitation, Vol. 21, No. 5, 2007, pp. 387-394. doi:10.1177/0269215507074055

[21] K. O. Berg, S. L. Wood Dauphinee, J. I. Williams and B. Maki, "Measuring Balance in the Elderly: Validation of an Instrument," Canadian Journal of Public Health, Vol. 83, No. 2, 1992, pp. S7-S11.

[22] H. Mao, I. Hsueh, P. Tang, C. Sheu and C. Hsieh, "Analysis and Comparison of the Psychometric Properties of Three Balance Measures for Stroke Patients," Stroke, Vol. 33, 2002, pp. 1022-1027. doi:10.1161/01.STR.0000012516.63191.C5

[23] J. Y. Wee, H. Wong and A. Palepu, "Validation of the Berg Balance Scale as a Predictor of Length of Stay and Discharge Destination in Stroke Rehabilitation," Archives of Physical Medicine and Rehabilitation, Vol. 84, No. 5, 2003, pp. 731-735.

[24] L. Blum and N. K. Bitensky, "Usefulness of Berg Balance Scale in Stroke Rehabilitation. A Systematic Review," Physical Therapy, Vol. 88, No. 5, 2008, pp. 559-566. doi:10.2522/ptj.20070205

[25] J. Green, A. Forster and J. Yong, "Reliability of Gait Speed Measured by a Timed Walking Test in Patients One Year after Stroke," Clinical Rehabilitation, Vol. 16, 
No. 3, 2002, pp. 306-314. doi:10.1191/0269215502cr495oa

[26] L. S. Portney and M. P. Watkins, "Power and Sample Size," In: L. S. Portney and M. P. Watkins, Ed., Foundations of Clinical Research: Applications to Practice, 2nd Edition, Prentice Hall, New Jersey, pp. 705-720.

[27] L. J. Liaw, C. L. Hsieh, S. K. Lo, H. M. Chen, S. Lee and J. H. Lin, "The Relative and Absolute Reliability of Two Balance Performance Measures in Chronic Stroke Patients," Disability and Rehabilitation, Vol. 30, No. 9, 2008, pp. 656-661. doi:10.1080/09638280701400698

[28] L. Alzayer, M. Beninato and L. G. Portney, "The Accuracy of Individual Berg Balance Scale Items Compared with the Total Berg Score for Classifying People with Chronic Stroke According to Fall History," Journal of Neurological Physical Therapy, Vol. 33, No. 3, 2009, pp. 136-143. doi:10.1097/NPT.0b013e3181b51307

[29] C. M. Dean and R. B. Shepherd, "Task Related Training Improves Performance of Seated Reaching Tasks after Stroke," Stroke, Vol. 28, 1997, pp. 722-728. doi:10.1161/01.STR.28.4.722

[30] C. M. Dean, E. F. Channon and J. M. Hall, "Sitting Training Early after Stroke Improves Sitting Ability and Quality and Carries over to Standing up but not to Walking: A Randomized Controlled Trial," Australian Journal of Physiotherapy, Vol. 53, No. 2, 2007, pp. 97-102. doi:10.1016/S0004-9514(07)70042-9

[31] J. K. Tilson, K. J. Sullivan, D. K. Cen, C. H. Koradia, S. P. Azen and P. W. Duncan, "Meaningful Gait Speed Im- provement during the First 60 Days Poststroke: Mimimal Clinically Important Difference," Physical Therapy, Vol. 90, No. 2, 2010, pp. 196-208. doi:10.2522/ptj.20090079

[32] P. A. Goldie, T. A. Matyas and O. M. Evans, "Gait after Stroke: Initial Deficit and Changes in Temporal Patterns for each Gait Phase," Archives of Physical Medicine and Rehabilitation, Vol. 82, No. 8, 2001, pp. 1057-1065. doi:10.1053/apmr.2001.25085

[33] J. C. Wall and G. I. Turnbull, "Gait Asymmetries in Residual Hemiplegia," Archives of Physical Medicine and Rehabilitation, Vol. 67, No. 8, 1983, pp. 550-553.

[34] J. W. van Nes, B. Nienhuis, H. Latour and A. C. Geurtus, "Posturographic Assessment of Sitting Balance Recovery in the Sub-Acute Phase of Stroke," Gait Posture, Vol. 28, No. 3, 2008, pp. 507-512. doi:10.1016/j.gaitpost.2008.03.004

[35] J. S. Chern, C. Y. Lo, C. Y. Wu, C. L. Chen, S. Yang and F. D. Tang, "Dynamic Postural Control during Trunk Bending and Reaching in Healthy Adults and Stroke Patients," American Journal of Physical Medicine and Rehabilitation, Vol. 89, No. 3, 2010, pp. 186-197. doi:10.1097/PHM.0b013e3181c56287

[36] S. F. Tyson, "Trunk Kinematics in Hemiplegic Gait and Effect of Walking Aids," Clinical Rehabilitation, Vol. 13, No. 4, 1999, pp. 295-300. doi:10.1191/026921599666307333

[37] P. R. Trueblood, J. M. Walker, J. Perry and J. K. Gronley, "Pelvic Exercise and Gait in Hemiplegia," Physical Therapy, Vol. 69, No. 1, 1989, pp. 18-26. 